\title{
A review of David F. Bjorklund's How Children Invented Humanity: The Role of Development in Human Evolution
}

\author{
New York: Oxford University Press, 2020, 392 pp., ISBN-13: 978-0190066864
}

\author{
Farid Pazhoohi ${ }^{1}$
}

Accepted: 15 October 2021 / Published online: 26 October 2021

(c) The Author(s), under exclusive licence to Springer Nature Switzerland AG 2021

David F. Bjorklund, Professor of Psychology at Florida Atlantic University, has influentially contributed to the development of evolutionary developmental psychology as a field. The general argument of Bjorklund in How Children Invented Humanity: The Role of Development in Human Evolution is that ontogeny has shaped the evolution of humans. He argues that the changes in development of our ancestors (e.g., during infancy and childhood) over the course of evolution have resulted in the phylogenetic changes differentiating us as a unique species. In his own words, "evolution, or phylogeny, can be viewed as a series of successive ontogenies, and it was changes in these ontogenies over generations, brought about by genetic mutations, shifts in gene expression, or alterations in the physical or social environments, that produced adults, who in turn reproduced more of their kind who continued the developmental and evolutionary process."

Bjorklund explains that by possessing substantial plasticity in early life, brains of young animals including human infants and children coordinate development between neural maturation, perceptual experience, and gene expression, adapting to a wide range of environmental conditions. Humans evolved a unique plasticity capacity, sensitive to environmental conditions early in development, which enables children to get prepared for exposure to all possible environments across the world, assuming children's early life conditions are good predictors of their adult life conditions. He argues that the behavioral variations in responsiveness to the environments are subject to natural selection, thereby development has played an important role in the evolution of our species.

Furthermore, through introducing the field of evolutionary developmental biology (Evo Devo), Bjorklund emphasizes

Farid Pazhoohi

pazhoohi@gmail.com

1 Department of Psychology, University of British Columbia, 2136 West Mall, Vancouver, British Columbia V6T 1Z4, Canada neoteny in humans (retention of juvenile traits into adulthood), arguing how neoteny has resulted in temporal extension of brain development in humans and retaining substantial levels of plasticity throughout life. From a comparative perspective, Bjorklund shows how humans are physically, behaviorally, and cognitively neotenous compared to other apes, highlighting how cognitive neoteny has been adaptive, playing a critical role in human evolution and development.

Bjorklund also focuses on the importance of childhood play in development of socio-cognitive abilities necessary for efficient functioning as adults of a hypersocial species. To Bjorklund, humans' hypersociality is a result of evolution of social-cognitive abilities which are the consequence of changes during the early years of development.

Finally, Bjorklund discusses the mismatch between the environments in which our ancestors evolved in and the current ones we are living today, by focusing on adolescents and children and their prolongation of dependency, widespread use of social media, and hyper-individualism compared to ancestral childhood and adolescence.

How Children Invented Humanity is a very clear, easy, and interesting read. I not only highly recommend this to the students and researchers of psychological, biological, and anthropological sciences, but this book is also valuable to parents and to-be-parents to understand and learn how early developmental years shape and affect all aspects of their children's childhood, adolescence, and adulthood. Additionally, this is a well-referenced book on evolutionary developmental psychology and biology, providing a great review of the literature, covering lots of experiments and studies, combined with Bjorklund's deep understanding of evolutionary biology and great sense of narration. This is a joyful read!

Publisher's Note Springer Nature remains neutral with regard to jurisdictional claims in published maps and institutional affiliations. 